\title{
MARC BLOCH : DIX ANS APRÈS
}

Le 16 juin 1944, il y a maintenant dix ans très exactement une voiture s'arrêtait en pleine campagne, sur le chemin qui mène de Trévoux à Saint-Didier de Formans. - Juin : la prairie de Saône vivait sa grande splendeur de tous les étés. Les hautes graminées inclinaient leurs panaches sous la brise. De partout semblait sourdre la fécondité. Sur l'ordre des Allemands, vingt-six patriotes français descendirent de la voiture. On les fit entrer, deux par deux, dans un pré enclos de haies. Quelques pas. Une rafale. Le couple s'abimait, face contre terre. "Aux deux suivants!"....

Parmi ces hommes, Marc Bloch. Il y a dix ans.

$$
*^{*} *
$$

On me dit parfois : "Que pensez-vous qu'il subsiste de son ceuvre personnelle?" Porte ouverte à des débats byzantins. Il reste le talent, d'abord, qui est la marque même de la personnalité. Il reste tant d'excitations à comprendre et à faire comprendre, tant d'interprétations ingénieuses et solides, tant de suggestions et d'hypothèses à confirmer ou à infirmer. Ceci ôté, la matière même de son histoire s'est fondue dans un trésor commun. Comme il arrive toujours. D'une ouvre de grand historien persistent des structures, des hypothèses de travail fécondes, des jalonnements de voies nouvelles. La lettre de ce qu'il a écrit? Il est rare qu'elle survive intacte pendant de longues années. Voyez l'œuore de Pirenne. Elle vit dans la mesure même où chacune de ses grandes vues de génie a provoqué les recherches d'une dizaine d'historiens qui la grignotent, la rectifient partiellement, l'épluchent et l'échenillent - et font ainsi qu'audessus d'eux elle vit toujours et s'impose. Quand le menu peuple 
des amateurs d'histoire aura cessé, une bonne fois, de confondre l'exactitude scolaire (c'est-à-dire la conformité avec une vulgate officielle) avec la vérité créatrice, un grand pas sera fait vers l'intelligence.

Que reste-t-il de vivant, que reste-t-il d'intact de l'œuvre personnelle de Marc Bloch? Mais tout sur le plan de la recherche. Tout ce que contient d'indications à la fois précieuses et sobres son "gros enfant", comme il disait - ses Rois thaumaturges. Dont tous les enseignements n'ont point été tirés. Tout ce qu'a provoqué d'études et de recherches, de contradictions et de confirmations ce classique, Les Caractères originaux, qui à la lucidité d'une exposition faite oralement, joint les richesses d'une investigation sans répit ni défaillances. Tout ce qui anime enfin d'un souffle de vie neuve les deux polumes de sa Civilisation médiévale, c'est-à-dire les deux pièces de son ouore qu'il m'est le plus difficile de juger, parce que c'est en elles que s'affirme le mieux sans doute l'action réciproque que, par tant de contacts immédiats et directs, nous avons pendant des années exercée l'un sur l'autre... - Mais poser la question ainsi c'est la mal poser. Il faut, pour être utile et suivi, la renverser, et dire : "Qu'avons-nous apporté, ami disparu et toujours si présent - qu'avons-nous apporté, nous qui sommes restés debout, derrière vous - à l'œusre commune, et qui l'ait enrichie, et dont nous puissions aujourd'hui pous faire hommage?"

Je n'ai guère à répondre qu'une chose : "Les Annales continuent." Nos Annales. Avec sans doute un grand vide toujours. Un vide que personne ne saurait combler. Mais quant à l'essentiel? Histoire agraire; histoire monétaire; histoire des prix; histoire des mythes, dans un autre domaine; histoire des incidences de l'économie sur la vie culturelle; histoire des classes sociales, etc. : tout ce que Marc Bloch a touché de sa main, marqué de son empreinte et, si souvent, je puis le dire, de notre commune empreinte - tout cela, nous avons travaillé à le développer, à le vivifier, à le mobiliser. Et nous pouvons crier : Partie gagnée! - Non pas que les résistances à l'œuvre des Annales aient cessé. Au contraire, on pourrait dire parfois qu'elles tendent à se faire plus aigres, plus virulentes. Le climat de 1954 n'est plus celui de 1944. Bien des complicités d'évé- 
nements et d'idées, qui nous aidaient alors à franchir les obstacles, ont changé de signe et joueraient plutôt contre nous, si nous n'étions pas soutenus, en France el à l'étranger, par une telle poussée des esprits. La ruée même des jeunes vers l'enseignement secondaire et supérieur; la grande invasion des lycées et des facultés par ces régiments de novices qu'il faut instruiretout cela a eu pour conséquence de scolariser encore davantage l'histoire, les études d'histoire dans la France d'aujourd'hui. Et cette sorte de scolarisation n'est pas précisément faite pour nous réjouir. - Il est vrai. Mais non moins vrai aussi qu'on peut continuer aujourd'hui, sur ce plan, à nous ignorer, à ne pas rencontrer nos itinéraires ; qu'ils fussent mal tracés, qu'ils dussent nous conduire à une impasse, personne ne l'a prétendu ni ne saurait le prétendre. La roue tourne, lentement. De sa lente rotation les hommes, dérangés dans leurs habitudes, fidèles aux traditions même périmées, même nocives, peuvent bien se plaindre. Il n'est pas en leur pouvoir de l'interrompre. Pendant un temps peut-être, ils feront de l'"Histoire Annales» en vilipendant les Annales, ces empêcheuses de dormir en rond. C'est humain. C'est préva. Et puis après? Nous n'avons pas fait notre œuore, les uns et les autres, dans l'espoir d'une récompense, d'une gratitude commune de nos contemporains. Notre cuure : elle n'irriterait pas tant certains de nos adversaires, si malgré leur résistance elle ne s'imposait déjà à leur conformisme.

Oui, la roue tourne. Elle ne s'est pas arrêtée en juin 1944, le 16. Elle continue de nous entraîner tous, de l'avant, dans une histoire élargie aux limites d'une planète. Plus élargie sans doute que ne l'imaginait Marc Bloch. Mais de cette extension comme il eût été heureux! Il aurait trouvé en elle, une fois de plus, la justification de son sacrifice. - Et de son labeur?Non. Le labeur n'a pas besoin de justification. On l'aime.

Lucien Febvre 